\title{
Hydrogen atom in momentum space with a minimal length
}

\author{
Djamil Bouazit* and Nourredine Ferkous \\ Laboratoire de Physique Théorique (LPTh), Université de Jijel, \\ Boîte Postale 98, Ouled Aissa, 18000 Jijel, Algeria.
}

\begin{abstract}
A momentum representation treatment of the hydrogen atom problem with a generalized uncertainty relation, which leads to a minimal length $\left(\Delta X_{i}\right)_{\min }=\hbar \sqrt{3 \beta+\beta^{\prime}}$, is presented. We show that the distance squared operator can be factorized in the case $\beta^{\prime}=2 \beta$. We analytically solve the s-wave bound-state equation. The leading correction to the energy spectrum caused by the minimal length depends on $\sqrt{\beta}$. An upper bound for the minimal length is found to be about $10^{-9}$ fm.
\end{abstract}

*Electronic address: djamilbouaziz@mail.univ-jijel.dz 


\section{INTRODUCTION}

Since Kempf and co-workers developed the theoretical framework of quantum mechanics based on a generalized uncertainty relation, which implies the existence of a minimal length, in a series of papers [1 3], a lot of attention has been attracted to the study of physical problems within this formalism, see, for instance, Refs. [4-13]. The idea of modifying the standard Heisenberg uncertainty relation in such a way that it includes a minimal length has first been proposed in the context of quantum gravity and string theory [14,15]. It is assumed that this elementary length should be on the scale of the Planck length of $l_{p}=10^{-35} \mathrm{~m}$, below which the resolution of distances is impossible.

It was shown in Refs. [1-3] that the minimal length uncertainty relation is closely connected to a modification of the standard Heisenberg algebra by adding specific corrections to the canonical commutation relations between position and momentum operators, so that the Heisenberg algebra becomes $\left[\widehat{X}_{i}, \widehat{P}_{j}\right]=i \hbar\left[\left(1+\beta \widehat{P}^{2}\right) \delta_{i j}+\beta^{\prime} \widehat{P}_{i} \widehat{P}_{j}\right]$, where $\beta$ and $\beta^{\prime}$ are small positive parameters related to the minimal length by $\left(\Delta X_{i}\right)_{\min }=\hbar \sqrt{3 \beta+\beta^{\prime}}$. One of the fundamental consequences of the generalized uncertainty relation is the loss of localization in coordinate space due to the presence of a nonzero minimal uncertainty in position measurements. Consequently, momentum space is more convenient in order to solve any eigenvalue problem. However, this is not often possible, especially when the potential depends, in a not too straightforward manner, on the position operators as in the case of the hydrogen atom potential. In the literature, this problem is the most studied in this modified version of quantum mechanics [16-20]. This is natural because this system has a particular interest. The elementary length has been associated to with finite size of the electron; and the use of the high-precision experimental data for the transition $1 S-2 S$ and for the Lamb shift were exploited to estimate an upper bound for the minimal length of about $0.01-0.1 \mathrm{fm}$.

Except in Ref. [16], the energy spectrum of the hydrogen atom has been obtained perturbatively in coordinate space: The terms proportional to the deformation parameters $\beta$ and $\beta^{\prime}$ in the Schrödinger equation were regarded as perturbation corrections to the Hamiltonian operator; the use of perturbation theory allowed for the computation of the corrections in the first order to the energy levels. In momentum space, the difficulty lies in defining the square root of the operator $\widehat{R}^{2}=\sum_{i=1}^{3} \widehat{X}_{i} \widehat{X}_{i}$. To avoid this problem, the author of Ref. [16] used complicated successive transformations on the wave function and solved the $s$-wave 
bound-state equation. The spectrum is obtained, however, the correction caused by the minimal length is different from what was obtained in coordinate spaces.

Given the discrepancy between the results, which concern this problem, it is interesting to consider it again with another method. For this purpose, here, we give a simple method to solve the $s$-wave deformed Schrödinger equation in momentum space for the Coulomb potential. We show that, in the particular case $\beta^{\prime}=2 \beta$, the distance squared operator $\widehat{R}^{2}$ can be factorized in the first order in the deformation parameter $\beta$. We obtain the wave function and the energy spectrum, which are different from that of Ref. [16]. By using the experimental data for the Lamb shift, we find an upper bound of the minimal length of $10^{-9}$ fm.

The rest of this paper is organized as follows. In Sec. II, we give a brief review of different works, which concern the hydrogen potential with a minimal length. In Sec. III, we study this problem in momentum space. We summarize our results in a brief concluding section.

\section{HYDROGEN ATOM WITH A MINIMAL LENGTH: A REVIEW}

As mentioned in Sec. I, several papers have been devoted to the study of the hydrogen atom problem in quantum mechanics with a generalized uncertainty relation, based on the following deformed Heisenberg algebra [16-20]:

$$
\begin{aligned}
{\left[\widehat{X}_{i}, \widehat{P}_{j}\right] } & =i \hbar\left[\left(1+\beta \widehat{P}^{2}\right) \delta_{i j}+\beta^{\prime} \widehat{P}_{i} \widehat{P}_{j}\right] \\
{\left[\widehat{P}_{i}, \widehat{P}_{j}\right] } & =0 \\
{\left[\widehat{X}_{i}, \widehat{X}_{j}\right] } & =i \hbar \frac{2 \beta-\beta^{\prime}+\beta\left(2 \beta+\beta^{\prime}\right) \widehat{P}^{2}}{1+\beta \widehat{P}^{2}}\left(\widehat{P}_{i} \widehat{X}_{j}-\widehat{X}_{i} \widehat{P}_{j}\right) .
\end{aligned}
$$

Many representations of the operators $\widehat{X}_{i}$ and $\widehat{P}_{i}$ were used by assuming that $\hat{X}_{i}$ and $\hat{P}_{i}$ are functions of the operators $\hat{x}_{i}$ and $\hat{p}_{i}$, which satisfy the standard canonical commutation relations of ordinary quantum mechanics.

Brau was the first to use the perturbation technique to calculate the correction to the energy spectrum of the hydrogen atom due to the presence of a minimal length [17]. The author made a simple choice of $\hat{X}_{i}$ and $\hat{P}_{i}$ in the coordinate space valid in the case $\beta^{\prime}=2 \beta$, and in the first order in $\beta$, namely

$$
\widehat{X}_{i}=\widehat{x}_{i}, \quad \widehat{P}_{i}=\widehat{p}_{i}\left(1+\beta \widehat{p}^{2}\right) .
$$


Thus, the Schrödinger equation takes the form

$$
\left(\frac{\widehat{p}^{2}}{2 m}+V(\widehat{\vec{r}})+\frac{\beta}{m} \widehat{p}^{4}\right) \psi(\vec{r})=E \psi(\vec{r}) .
$$

As is clearly seen, the effect of the minimal length is described by the presence of a perturbation term $\left(\frac{\beta}{m} \widehat{p}^{4}\right)$ in the ordinary Schrödinger equation.

Thereafter, Akhoury and Yao [16] considered the same problem in momentum space by using the following representation:

$$
\widehat{X}_{i}=\left(1+\beta \widehat{p}^{2}\right) \widehat{x}_{i}+\beta^{\prime} \widehat{p}_{i} \widehat{p}_{j} \widehat{x}_{j}+\gamma \widehat{p}_{i}, \quad \widehat{P}_{i}=\widehat{p}_{i}
$$

The authors write the Schrödinger equation for the Coulomb potential $V(r)=-\alpha / r$, in the form

$$
\left(\widehat{R}\left(\frac{\widehat{p}^{2}}{2 m}-E\right)-\alpha\right)|\psi\rangle=0,
$$

where $\widehat{R}$ is the square root of the operator $\widehat{R}^{2}=\sum_{i=1}^{3} \widehat{X}_{i} \widehat{X}_{i}$.

Unlike in ordinary quantum mechanics, where the expression of $\widehat{R}$ can be obtained for the $s$-waves $(l=0)$, the definition of this operator is not obvious in the deformed case even if $l=0$.

To overcome this problem, Akhoury and Yao performed some changes of variables and transformations on the wave function, and defined a supposedly radial distance operator $\widehat{R}=$ $i \hbar\left[1+\left(\beta+\beta^{\prime}\right) p^{2}\right] \frac{d}{d p}$, which acts on the state $\tau|\psi\rangle$ instead of $|\psi\rangle$, where $\tau$ is a transformation not explicitly given. Nevertheless, the authors succeeded to get a solution to the deformed Schrödinger equation, and to extract the energy spectrum by imposing the condition of single valuedness on the wave function. The correction to the energy levels is completely different from that obtained by Brau. It is important to mention that in Ref. [16], the condition of single valuedness was not correctly applied. In Sec. III, we propose another method in momentum space; the correct energy spectrum will be calculated.

The problem of the hydrogen atom has been reconsidered by Benczik et al. [18], by using the representation given by Eq. (66) with two approaches, the first by numerical techniques in momentum space and the second by the perturbation theory in position space. Their results are in disagreement with those obtained by Akhoury and Yao, and differ from the ones of Brau only for $\ell=0$. 
Finally, Stetsko and Tkachuk [19] and [20] proposed another perturbative method by using the following position representation of the operators $\widehat{X}_{i}$ and $\widehat{P}_{i}$ :

$$
\widehat{X}_{i}=\widehat{x}_{i}+\frac{2 \beta-\beta^{\prime}}{4}\left(\widehat{p}^{2} \widehat{x}_{i}+\widehat{x}_{i} \widehat{p}^{2}\right), \quad \widehat{P}_{i}=\widehat{p}_{i}\left(1+\frac{\beta^{\prime}}{2} \widehat{p}^{2}\right) .
$$

This representation reduces to that of Brau Eq. (41) in the case $\beta^{\prime}=2 \beta$. They compute the correction to the energy spectrum in the first order in $\beta$ and $\beta^{\prime}$. Their results reproduce those of Brau even in the case $\ell=0$.

For the sake of completeness, let us mention that the Coulomb potential has also been considered in the one-dimensional case in both nonrelativistic [21] and relativistic [22] quantum mechanics with a minimal length. The treatment was performed in momentum space, and the expression of the energy spectrum is different from that obtained in the case $\ell=0$ of Ref. [16].

In the following, we consider, again, the hydrogen atom problem by using a momentum representation, which is more appropriate in this version of quantum mechanics.

\section{MOMENTUM SPACE TREATMENT}

Let us consider the Schrödinger equation for the hydrogen atom in the form

$$
\left(\widehat{R}\left(\frac{\widehat{p}^{2}}{2 m}-E\right)-\alpha\right)|\psi\rangle=0
$$

where the strength of the potential is $\alpha=\frac{e^{2}}{4 \pi \epsilon_{0}}$. In the momentum representation, the wave function reads [4]

$$
\psi(\vec{p})=\langle\vec{p} \mid \psi\rangle=Y_{\ell}^{m}(\theta, \varphi) \psi(p)
$$

By restricting ourselves to the $\ell=0$ wave function and by using the momentum representation given by Eq. (6), with $\gamma=0$, we obtain the following expression for the distance squared operator :

$$
\widehat{R}^{2}=(i \hbar)^{2}\left\{\left[1+\left(\beta+\beta^{\prime}\right) p^{2}\right]^{2} \frac{d^{2}}{d p^{2}}+\frac{2}{p}\left[1+\left(\beta+\beta^{\prime}\right) p^{2}\right]\left[1+\left(2 \beta+\beta^{\prime}\right) p^{2}\right] \frac{d}{d p}\right\} .
$$

In the general case, this operator is not factorizable in the sense that its square root is unknown. In spite of this, we show that $\widehat{R}^{2}$ can be factorized in the particular case $\beta^{\prime}=2 \beta$ in the first order in $\beta$. Indeed, since $\beta$ and $\beta^{\prime}$ are supposed to be small parameters, the 
distance squared operator can be expressed as

$$
\widehat{R}^{2}=(i \hbar)^{2}\left\{\left(1+6 \beta p^{2}\right) \frac{d^{2}}{d p^{2}}+\frac{2}{p}\left(1+7 \beta p^{2}\right) \frac{d}{d p}\right\}+O\left(\beta^{2}\right) .
$$

In Eq. (10), $\widehat{R}^{2}$ can be written as $\widehat{R} \times \widehat{R}$, where

$$
\widehat{R}=i \hbar\left[\left(1+3 \beta p^{2}\right) \frac{d}{d p}+\frac{1}{p}\left(1+\beta p^{2}\right)\right]+O\left(\beta^{2}\right) .
$$

From Eqs. (8) and (11), the radial Schrödinger equation for the hydrogen atom in momentum space with a minimal length reads

$$
\left(1+3 \beta p^{2}\right)\left(p^{2}+k^{2}\right) \frac{d \psi(p)}{d p}+\left\{\frac{1}{p}\left(1+\beta p^{2}\right)\left(p^{2}+k^{2}\right)+2 p\left(1+3 \beta p^{2}\right)+\frac{2 i \alpha m}{\hbar}\right\} \psi(p)=0,
$$

where $k^{2}=-2 m E$.

In order to integrate this equation, it is convenient to make it in the form

$$
\frac{d \psi(p)}{d p}+\left\{\frac{\eta-1}{p+i k}-\frac{\eta+1}{p-i k}+\frac{\frac{1}{3}-\xi}{p+i / \sqrt{3 \beta}}+\frac{\frac{1}{3}+\xi}{p-i / \sqrt{3 \beta}}-\frac{1}{p}\right\} \psi(p)=0,
$$

in which,

$$
\xi=\frac{\alpha m \sqrt{3 \beta}}{\hbar\left(1-3 \beta k^{2}\right)}, \quad \eta=\frac{\alpha m}{\hbar k\left(1-3 \beta k^{2}\right)} .
$$

The solution to Eq. (13) is

$$
\psi(p)=A \frac{\left(1+3 \beta p^{2}\right)^{1 / 3}}{p\left(p^{2}+k^{2}\right)} \exp [2 \xi i \arctan (p \sqrt{3 \beta})-2 \eta i \arctan (p / k)],
$$

where $A$ is a normalization constant. In the limit $\beta=0, \psi(p)$ reduces to the result of ordinary quantum mechanics [24].

Our wave function differs from that obtained in Ref. [16] by the factor $\frac{1}{p}\left(1+3 \beta p^{2}\right)^{1 / 3}$ :

$$
\psi(p)=\frac{1}{p}\left(1+3 \beta p^{2}\right)^{1 / 3} \psi(p)_{\text {Akhoury }}
$$

This discrepancy is due to a certain transformation used in Ref. [16], which has not been explicitly given.

To extract the energy spectrum, by following Refs. [16, 23-26], we require that $\psi(p)$ must be a single-valued function ( i.e., it must be unchanged under the transformation):

$$
\arctan (z) \rightarrow \arctan (z)+\pi
$$


Thus, we must have

$$
\xi-\eta=n,
$$

where $n$ is an integer number. This leads to the following quantization condition :

$$
\frac{\alpha m}{\hbar k(1+k \sqrt{3 \beta})}=n, \quad n=1,2, \ldots .
$$

By solving for $k$ and by using $k=\sqrt{-2 m E}$, we obtain

$$
E_{n}^{ \pm}=-\frac{1}{24 m \beta}\left(1 \pm\left(1+4 \frac{m \alpha}{\hbar n} \sqrt{3 \beta}\right)^{1 / 2}\right)^{2} .
$$

In the limit $\beta \rightarrow 0, E_{n}^{+}$diverges. So, the energy spectrum reads

$$
E_{n}=-\frac{1}{24 m \beta}\left(1-\left(1+4 \frac{m \alpha}{\hbar n} \sqrt{3 \beta}\right)^{1 / 2}\right)^{2}, \quad n=1,2, \ldots
$$

To leading orders in the small parameter $\beta$, the spectrum can be expressed as follows:

$$
E_{n}=-\left(\frac{m \alpha^{2}}{2 \hbar^{2} n^{2}}-\frac{m^{2} \alpha^{3}}{\hbar^{3} n^{3}} \sqrt{3 \beta}+\frac{15}{2} \frac{m^{3} \alpha^{4}}{\hbar^{4} n^{4}} \beta\right)+O\left(\beta^{\frac{3}{2}}\right), \quad n=1,2, \ldots
$$

The first term represents the energy spectrum of ordinary quantum mechanics, while the second and third terms are the corrections brought about by the existence of a minimal length. As we see, our result coincides with that of the one-dimensional Coulomb potential [21], where the quantization condition has been derived by imposing the Hermiticity of the Hamiltonian.

The main feature of the spectrum Eq. (18) is the presence of a positive correction proportional to the minimal length $\left((\Delta X)_{\min }=\hbar \sqrt{3 \beta}\right)$, which is the leading correction. Previously, this term was omitted in Ref. [16]; the correction due to the modification of the Heisenberg algebra is negative, and is described only by the third term of Eq. (18). This is because the condition of the single valuedness was not strictly applied. The authors took, instead of Eq. (15), the condition $\eta=n$, which is not sufficient to assure that the wave function be single valued. In the perturbative treatment of the hydrogen atom [17 20], $\beta$ is the perturbation parameter, and, naturally the first-order correction is proportional to this deformation parameter.

This result is very important because it leads to an order of magnitude of the minimal length completely different from what was obtained in Refs. [16-20], where an upper bound for the minimal length was found to be about $0.01-0.1 \mathrm{fm}$. The estimation of this bound 
was mainly obtained by two methods. The first requires that the corrections to the spectrum due to the modification of the Heisenberg algebra are smaller than the experimental error on the value of the transition $1 S-2 S$ in the hydrogen atom [16, 17]. The second assumes that the effects of the minimal length are included in the gap between the theoretical and the experimental values of the Lamb shift for the hydrogen atom levels [18 20].

Indeed, we now use Eq. (18) to give a new constraint for the minimal length. Let us write, to leading orders in the small parameter $\beta$, the following relative shift:

$$
\frac{E_{2 S}-E_{1 S}}{E_{1 S}}=-\frac{3}{4}+\frac{m \alpha}{4 \hbar} \sqrt{3 \beta}-\frac{21}{16} \frac{m^{2} \alpha^{2}}{\hbar^{2}} \beta+O\left(\beta^{\frac{3}{2}}\right) .
$$

The $1 S-2 S$ energy splitting in the hydrogen atom is measured with an accuracy of $\varepsilon=1.8 \times 10^{-14}[27]$. If we attribute this error entirely to the minimal length correction (19), and by taking only the first dominant contribution intoaccount, we can write

$$
\varepsilon=\frac{m \alpha}{4 \hbar} \sqrt{3 \beta}+O(\beta)
$$

which gives the value $(\Delta X)_{\text {min }} \sim 5 \times 10^{-9}$ fm. This upper bound is much smaller than the one obtained in Refs. [16, 17]. This is due to the absence of the term proportional to $\sqrt{3 \beta}$ in these references.

It was noted in Ref. [18] that a better estimate for the minimal length is obtained by including its corrections in the Lamb shift. Thus, from Eq. (18), the difference $\left(L_{1 S}^{\exp }-L_{1 S}^{t h}\right)$ can be taken as

$$
\Delta E_{1 S}=\frac{m^{2} \alpha^{3}}{\hbar^{3} \eta^{3}} \sqrt{3 \beta}=h\left(L_{1 S}^{\exp }-L_{1 S}^{t h}\right) .
$$

Given $L_{1 S}^{t h}=8172,731(40) \mathrm{MHz}$ [28] and $L_{1 S}^{\exp }=8172,837(22) \mathrm{MHz}$ [29], we obtain the value $(\Delta X)_{\min } \sim 10^{-6} \mathrm{fm}$. Again, we have a stringent limit on the value of the minimal length. One can conclude that, in non relativistic treatment of the hydrogen atom problem, the inclusion of a minimal length would not affect the hydrogen atom physics because its predicted size is too small.

\section{SUMMARY}

We have proposed a simple method to solve the $s$-wave Schrödinger equation in momentum space for the hydrogen atom problem in the framework of quantum mechanics with a 
generalized uncertainty relation, characterized by a minimal length $(\Delta X)_{\min }=\hbar \sqrt{3 \beta+\beta^{\prime}}$. We have shown that the distance squared operator $\widehat{R}^{2}$ is factorizable in the case $\beta^{\prime}=2 \beta$ in the first order in the deformation parameter $\beta$. The wave functions and the corresponding energy levels are obtained. The leading correction to the energy spectrum is proportional to $\sqrt{\beta}$, which is in agreement with that of the one-dimensional case [21]. The dependence on $\sqrt{\beta}$ drastically lowers the minimal length scale, which is of about $10^{-9} \mathrm{fm}$. This leads us to conclude that the minimal length in the problem considered here is too small so that its effects on the hydrogen atom physics are negligible.

\section{Acknowledgments}

We thank Professor Tahar Boudjedaa for several very instructive discussions. This research is supported by the Algerian Ministry of Higher Education and Scientific Research under the CNEPRU Projects No D01720060022 and No D01720090023.

[1] A. Kempf, G. Mangano and R. B. Mann, Phys. Rev. D 52, 1108 (1995).

[2] A. Kempf, J. Phys. A: Math. Gen. 30, 2093 (1997).

[3] A. Kempf, J. Math. Phys. 35, 4483 (1994), H. Hinrichsen and A. Kempf, J. Math. Phys. 37, 2121 (1996).

[4] L. N. Chang, D. Minic, N. Okamura, and T. Takeuchi, Phys. Rev. D 65, 125027(2002).

[5] S. Benczik et al, Phys. Rev. D 66, 026003 (2002).

[6] F. Brau and F. Buisseret, Phys. Rev. D 74, 036002 (2006).

[7] D. Bouaziz and M. Bawin, Phys. Rev. A 76, 032112 (2007).

[8] U. Harbach and S. Hossenfelder, Phys. Lett. B 632, 379 (2006).

[9] Kh. Nouicer, J. Phys. A: Math. Gen. 38, 10027 (2005).

[10] D. Bouaziz and M. Bawin, Phys. Rev. A 78, 032110 (2008).

[11] A. Bina, S. Jalalzadeh and A. Moslehi, Phys. Rev. D 81, 023528 (2010).

[12] C. Quesne and V. M. Tkachuk, Phys. Rev. A 81, 012106 (2010).

[13] M. Falek and M. Merad, J. Math. Phys. 51, 033516 (2010).

[14] L. J. Garay, Int. J. Mod. Phys. A 10, 145 (1995). 
[15] D. Amati, M. Ciafaloni, and G. Veneziano, Phys. Lett. B 216, 41 (1989).

[16] R. Akhoury and Y. P. Yao, Phys. Lett. B 572, 37 (2003).

[17] F. Brau, J. Phys. A 32, 7691 (1999).

[18] S. Benczik, L. N. Chang, D. Minic, and T. Takeuchi, Phys. Rev. A 72, 012104 (2005).

[19] M. M. Stetsko and V. M. Tkachuk, Phys. Rev. A 74, 012101 (2006).

[20] M. M. Stetsko, Phys. Rev. A 74, 062105 (2006).

[21] T.V. Fityo, I.O. Vacarchuk, V.M. Tkachuk, J. Phys. A 39, 2143 (2006).

[22] Y. Chargui, A. Trabelsi and L. Chetouani, Phys. Lett. A 374, 2243 (2010).

[23] J. R. Lombardi, Phys. Rev. A 22, 797 (1980).

[24] E. V. Ivash, Am. J. Phys. 40, 1095 (1972).

[25] H.N. Nunez Yepez, C.A. Vargas and A.L.S. Brito, Eur. J. Phys. 8, 189 (1987).

[26] M. M. Nieto, Phys. Rev. A 29, 3413 (1984).

[27] M. Niering, et al., Phys. Rev. Lett. 84, 5496 (2000).

[28] S. Mallampalli and J. Sapirstein, Phys. Rev. Lett. 80, 5297 ( 1998).

[29] C. Schwob et al., Phys. Rev. Lett 82, 4960 (1999). 